\title{
Dark Matter Alternative Passes Big Test
}

\section{A cosmological model that doesn't require dark matter has overcome a major hurdle in matching observations from the cosmic microwave background.}

\author{
By Michael Schirber
}

$W$ esearchers pursuing an unconventional view of cosmology that dispenses with dark matter have developed a model that can match observations of the cosmic microwave background (CMB), the leftover glow of the big bang [1]. This dark-matter-free model is an extension of the so-called MOND (modified Newtonian dynamics) theory, which assumes that the gravitational force on galaxy scales is different from the standard Newtonian force. Previous MOND-based models could not reproduce the CMB. The researchers say that their model can be further tested with observations of galaxy clusters and gravitational waves.

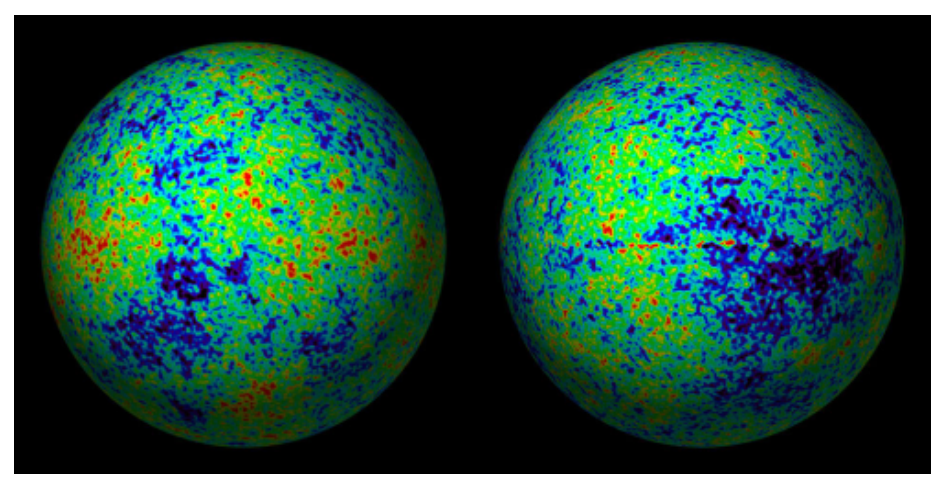

Sky patterns. MOND models-which don't require dark matter-have not previously been able to reproduce the temperature variations measured in the cosmic microwave background (shown here), a relic from the big bang. But now researchers have created a MOND-inspired model that matches these data just as well as dark matter models do.

Credit: WMAP Science Team/NASA
The MOND theory was devised more than 30 years ago as a way to explain galactic rotation data without invoking the existence of the mysterious dark matter [2]. MOND proponents offered an alternative mystery in which the gravitational force changes for accelerations smaller than a threshold of $10^{-10} \mathrm{~m} / \mathrm{s}^{2}$. The idea did not spring from any underlying theory, but surprisingly, the same acceleration threshold works for nearly all galaxies-small and large, young and old.

The main reason that dark matter has been favored over MOND is that dark matter is consistent with a much larger range of astrophysical observations. For example, dark matter can explain galaxies' bending of light from distant sources (gravitational lensing), whereas MOND in its initial form could not. Researchers have devised so-called relativistic MOND models that can fit the lensing observations [3], but until now, none of these revised versions of the theory were able to reproduce CMB data. "If the theory can't do that, then it's not worth considering further," says Constantinos Skordis from the Czech Academy of Sciences in Prague.

Skordis and Czech Academy colleague Tom Złośnik have now created a MOND-inspired model that accounts for the CMB while also being consistent with gravitational lensing observations and gravitational-wave speed measurements. The model follows recent MOND efforts in postulating the existence of two fields that permeate all of space and together act like an extra gravitational force. One of these fields is a scalar field-similar to the Higgs field that is associated with the Higgs boson. The other is a vector field, which has a direction at each point in space, somewhat like a magnetic field. 


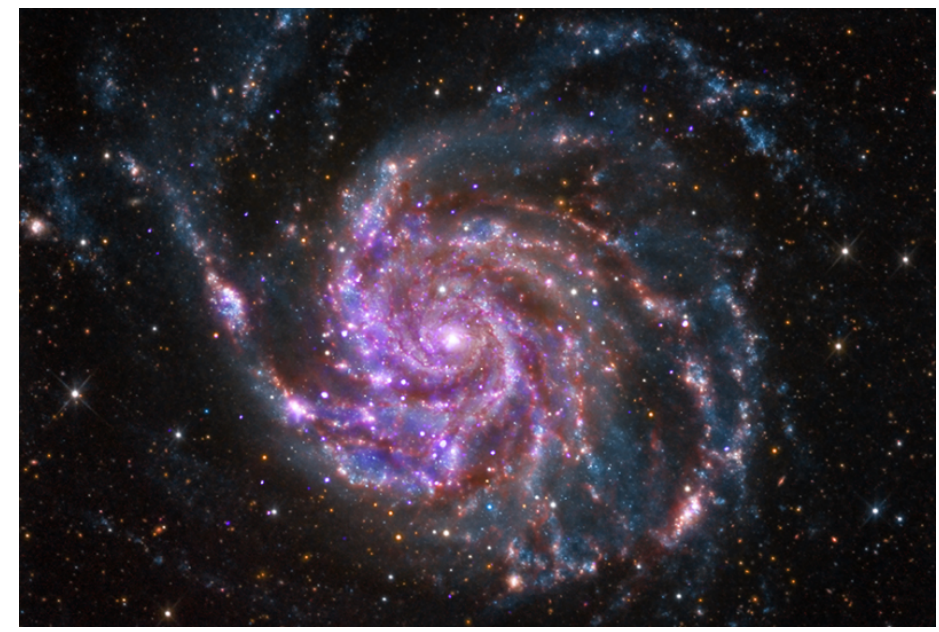

Turnaround is fair play. Galaxies, like M101 shown here, have rotation profiles that can't be explained by the visible matter. The popular solution is to assume the existence of dark matter, but another solution, called MOND, can account for galaxy data by modifying Newtonian gravity.

Credit: NASA/CXC/SAO/D. Hartmann/JPL-Caltech

Skordis and Ztośnik set the model's parameters so that, in the early Universe, the gravity-modifying fields generate a gravitational effect that mimics that of dark matter. Mimicking dark matter in this way ensures that the observed CMB patterns are reproduced. The fields evolve over cosmic time, and eventually the gravitational force follows the original MOND proposal.

Skordis says that the model is similar to other alternative gravity models that have been proposed to explain dark energy (see Viewpoint: Reining in Alternative Gravity). All cosmological models add something (new particles or new fields) to explain observations, he says. He admits that-unlike dark matter models that are often based on fundamental symmetry principles-the new model was not conceived with an underlying theory in mind. However, such a theoretical basis might be uncovered using the new MOND model.

"It really is a huge breakthrough," says cosmologist Stacy McGaugh from Case Western Reserve University in Ohio. "For years stretching into decades, people have largely ignored MOND because it seemed impossible to do what Skordis and Złośnik have now done." But David Spergel, a cosmologist from the Flatiron Institute in New York, finds the new model "baroque." He argues that relativistic MOND models only work by "effectively positing a complex form of dark matter."

Cosmologist Katherine Freese from the University of Texas congratulates the researchers for their accomplishment. "It is a big deal to construct a relativistic version of MOND that is able to match all existing data, especially fitting CMB data along with the MOND phenomenology in galaxies," she says. "However, the model has a lot of ingredients," she says. "I myself would still vote for dark matter as a simpler explanation."

McGaugh counters that dark matter models cannot explain everything, such as the Universe's lithium abundance or the discrepancies between different types of measurements of the cosmic expansion rate. The new MOND model might be able to solve these problems, but Skordis says that it will take more time to work out the theoretical details. He says that the model can be checked in other ways, for example, by comparing its predictions with observations of galaxy clusters or by looking for signatures of the gravity-modifying fields in gravitational waves.

Tessa Baker, an expert in alternative gravity models from Queen Mary University of London says that if dark matter detectors continue to come up empty, "then we may see increased interest in this family of modified gravity models."

Michael Schirber is a Corresponding Editor for Physics based in Lyon, France.

\section{REFERENCES}

1. C. Skordis and T. Złośnik, "New relativistic theory for modified Newtonian dynamics," Phys. Rev. Lett. 127, 161302 (2021).

2. M. Milgrom, "On the use of galaxy rotation curves to test the modified dynamics," Astrophys. J. 333, 689 (1988).

3. J. D. Bekenstein, "Relativistic gravitation theory for the modified Newtonian dynamics paradigm," Phys. Rev. D 70, 083509 (2004). 